\title{
Fungal phylogenies and plant functional traits structure root associated fungal networks in a subtropical forest
}

\author{
Chunchao Zhu ${ }^{1}$, Zihui Wang ${ }^{2}$, Wenqi Luo ${ }^{2}$, Jiayi Feng ${ }^{2}$, Yongfa Chen ${ }^{2}$, Dong $\mathrm{He}^{2}$, M. D. \\ Farnon Ellwood ${ }^{3}$, Chengjin $\mathrm{Chu}^{4}$, and Yuanzhi $\mathrm{Li}^{2}$ \\ ${ }^{1}$ Zunyi Medical University - Zhuhai Campus \\ ${ }^{2}$ Sun Yat-Sen University \\ ${ }^{3}$ Affiliation not available \\ ${ }^{4}$ Sun Yan-sen University of Medical Sciences
}

November 20, 2020

\begin{abstract}
Rhizosphere fungi are essential for plant survival and ecosystem functioning, but the processes structuring plant-fungal interactions remain largely unknown. We constructed association networks between 43 plant species and two groups of root-associated fungi (mycorrhizal and pathogenic) using sequence data. We revealed modularity within the association networks using network analysis, and correlated this modular structure with functional traits and phylogenetic history driving plant-fungal interactions. We observed strong modularity in both plant-mycorrhizal fungal and plant-pathogenic fungal association networks. Plant functional traits and fungal phylogeny clustered within modules. Host plants of mycorrhizal fungi differed significantly between modules in terms of their leaf dry matter content, photosynthetic traits and root tissue density. Host plants of pathogenic fungi differed significantly between modules in terms of their dark respiration rate, light compensation point and root morphology. Modularity within fungi was a product of fungal phylogeny, whereas host plant modularity was a product of functional traits (leaf morphology, photosynthetic rate and root morphology). Our study illustrates the link between plant functional traits and fungal assembly, and highlights the importance of niche-based processes in shaping plant-fungus association networks. Our results suggest that plant traits may be instrumental in managing the composition of belowground fungal communities.
\end{abstract}

\section{Hosted file}

Main document-ori.pdf available at https://authorea.com/users/377311/articles/494031-fungalphylogenies-and-plant-functional-traits-structure-root-associated-fungal-networks-in-asubtropical-forest 
(a) Modularity of plant-MF association network

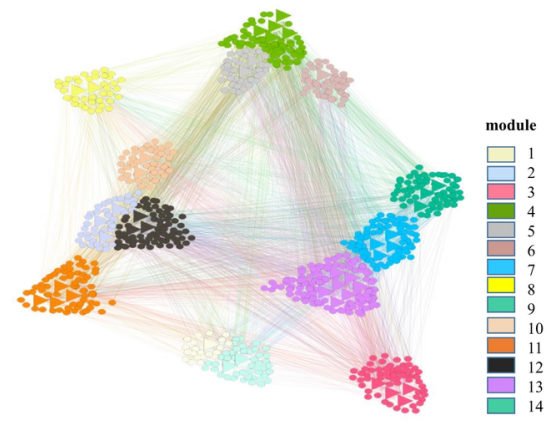

Relative modularity $=\mathbf{7 . 3 3}$ (b) Modularity of plant-PF association network

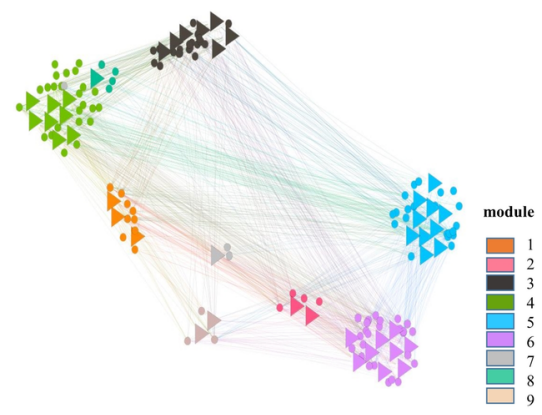

Relative modularity $=\mathbf{9 . 1 7}$
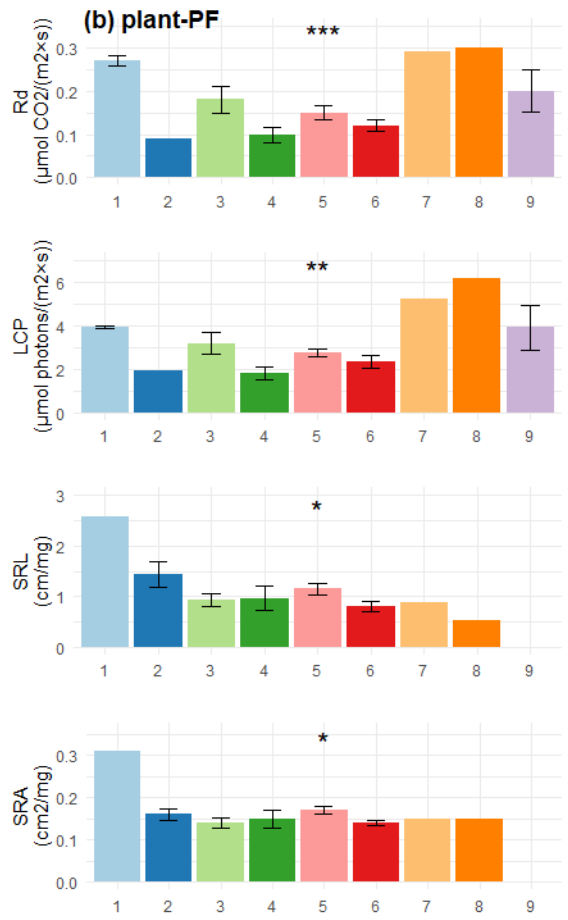
(a) Module plants in plant-MF association network

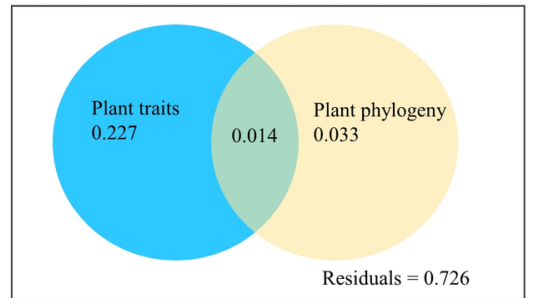

(c) Module MF in plant-MF association network

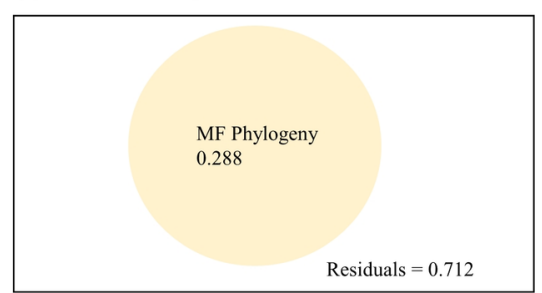

(b) Module plants in plant-PF association network

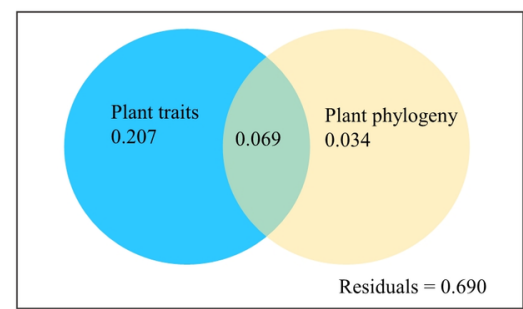

(d) Module PF in plant-PF association network

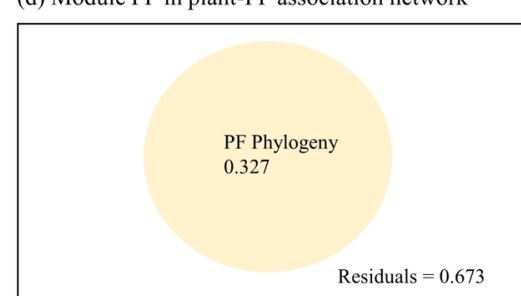

